\title{
Crashes, Recoveries, and 'Core-shifts' in a Model of Evolving Networks
}

\author{
Sanjay Jain ${ }^{*, \dagger, \ddagger, \uparrow}$ and Sandeep Krishna* \\ ${ }^{*}$ Centre for Theoretical Studies, Indian Institute of Science, Bangalore 560 012, India \\ $\dagger^{\dagger}$ Santa Fe Institute, 1399 Hyde Park Road, Santa Fe, NM 87501, USA \\ $\ddagger$ Jawaharlal Nehru Centre for Advanced Scientific Research, Bangalore 560 064, India \\ 『 Emails: jain@cts.iisc.ernet.in, sandeep@physics.iisc.ernet.in
}

\begin{abstract}
A model of an evolving network of interacting molecular species is shown to exhibit repeated rounds of crashes in which several species get rapidly depopulated, followed by recoveries. The network inevitably self-organizes into an autocatalytic structure, which consists of an irreducible 'core' surrounded by a parasitic 'periphery'. Crashes typically occur when the existing autocatalytic set becomes fragile and suffers a 'core-shift', defined graph theoretically. The nature of the recovery after a crash, in particular the time of recovery, depends upon the organizational structure that survives the crash. The largest eigenvalue of the adjacency matrix of the graph is an important signal of network fragility or robustness.
\end{abstract}

PACS numbers: 89.75.Fb, 87.23.Kg, 64.60.Cn, 05.65.+b

The dynamics of crashes and recoveries has been the subject of several empirical and modeling studies in macroevolution (for reviews, see [1,2]) and finance [3, 5]. The main attempt of most models has been to reproduce quantitatively the observed statistics of event sizes. However, it is also worthwhile to ask whether large events share other common features, or signatures, that precede the event or characterize the kind of systemic transformation caused by them. Here we present a structural analysis, based on network properties, of events that occur in a model [6] in which populations of molecular species coevolve with their network of catalytic interactions [9]. We find large crashes to be associated with a particular kind of structural change in the network, which we call a 'core-shift', and identify network characteristics that signal the system's susceptibility to crashes. This kind of analysis might be useful for other models of biological and social evolution.

The system is a directed graph with $s$ nodes labeled $i \in S \equiv\{1,2, \ldots, s\}$, represented by its adjacency matrix $C \equiv\left(c_{i j}\right)$. If there exists a directed link from node $j$ to node $i$ in the graph then $c_{i j}=1$, else $c_{i j}=0$. Each node represents a molecular species in a prebiotic pond and $c_{i j}=1$ means that $j$ is a catalyst for the production of $i$. The dynamical variables are the 'relative population vector' of the species $\mathbf{x} \equiv\left\{\left(x_{1}, \ldots, x_{s}\right) \mid 0 \leq x_{i} \leq\right.$ $\left.1, \sum_{i=1}^{s} x_{i}=1\right\}$, which is a fast variable, and the graph itself (or $C$ ), which is a slow variable. Initially, each $c_{i j}$ for $i \neq j$ is independently chosen to be unity with a probability $p$ and zero with a probability $1-p$. To exclude self-replicating species, $c_{i i} \equiv 0$ for all $i$. Each $x_{i}$ is chosen randomly in $[0,1]$ and all $x_{i}$ are rescaled so that $\sum_{i=1}^{s} x_{i}=1$. With $C$ fixed, $\mathbf{x}$ is evolved according to

$$
\dot{x}_{i}=\sum_{j=1}^{s} c_{i j} x_{j}-x_{i} \sum_{k, j=1}^{s} c_{k j} x_{j}
$$

until it reaches its attractor (always a fixed point [6, 10]), denoted X. (1) is an idealization of rate equations for catalyzed reactions in a well stirred chemical reactor. The set $\mathcal{L}$ of nodes with the least $X_{i}$ is determined, i.e,
$\mathcal{L}=\left\{i \in S \mid X_{i}=\min _{j \in S} X_{j}\right\}$. A node, denoted $k$, is picked randomly from $\mathcal{L}$ and for every $i \neq k c_{i k}$ and $c_{k i}$ are independently reassigned to unity with probability $p$ and zero with probability $1-p$, irrespective of their earlier values. This corresponds to removing the node $k$ and all its links from the graph and replacing it by a new node $k$ with random links to and from the other nodes. $c_{k k}$ is set to zero, $x_{k}$ is set to a small constant $x_{0}$, all other $x_{i}$ are perturbed by a small amount from their existing value $X_{i}$, and all $x_{i}$ are rescaled so that $\sum_{i=1}^{s} x_{i}=1$. This captures, in an idealized way, the impact of a periodic fluctuation like a tide or flood, which can wash out one of the least populated species in the pond (extremal selection [11]), and bring in a new molecular species whose catalytic links with those in the pond are random (introduction of novelty). Then $\mathbf{x}$ is again evolved to its new attractor, another graph update is performed, and so on.

Fig. 1 shows the number of populated species in the attractor (i.e., species with $X_{i}>0$ ), $s_{1}$, as a function of time for three runs with different $p$ values. Time is represented by $n$, the number of graph updates. Three regimes or phases of behaviour can be observed. First, the 'random phase' in which $s_{1}$ fluctuates about a low value. Second, the 'growth phase', when $s_{1}$ shows a clear rising tendency (occasionally punctuated by drops). Third, the 'organized phase' where $s_{1}$ stays close to its maximum value, $s$. The average time spent in each phase depends upon $p$ and $s$. In this letter we investigate the large and sudden drops in $s_{1}$, visible in Fig. 1 (mentioned briefly in [12]). These 'crashes' in the organized and growth phases are followed by 'recoveries', in which $s_{1}$ rises on a certain timescale. Fig. 2 shows the probability distribution $P\left(\Delta s_{1}\right)$ of changes in the number of populated species, $\Delta s_{1}(n) \equiv s_{1}(n)-s_{1}(n-1)$. The asymmetry between rises and drops as well as fat tails in the distribution of fluctuations are evident. For low $p$ the probability of large drops is an order of magnitude greater than intermediate size drops (also see Fig. 1) 13].

An autocatalytic set (ACS) is said to be a set of species which contains a catalyst for each of its members $[14-16$. Here it is a subgraph each of whose nodes has at least 
one incoming link from a node of the same subgraph. (A subgraph is a subset of nodes together with all their mutual links.) For example in Fig. 3, the subgraph formed by nodes 40, 93, 36, 51 is not an ACS, but that formed by $40,93,36,51,63$ is. The subgraph of all black nodes is also an ACS. Let $\lambda_{1}(C) \equiv \lambda_{1}$ be the largest eigenvalue of $C$. It can be shown [10] that $(i)$ if the graph does not have an ACS then $\lambda_{1}=0$, and if it does then $\lambda_{1} \geq 1$. (ii) $\mathbf{X}$ is an eigenvector of $C$ with eigenvalue $\lambda_{1}$. (iii) The set of nodes for which $X_{i}>0$ is uniquely determined by $C$, independent of (generic) initial condition on $\mathbf{x}$. ( $i v)$ If $\lambda_{1} \geq 1$ the subgraph formed by the set in (iii) constitutes an ACS, which will be referred to as the 'dominant ACS'. (We find $\mathbf{X}$ from these algebraic properties, rather than numerically integrating (11).)

In the random phase the graph has no ACS (in Fig. 1 this phase coincides with $\lambda_{1}=0$ ). The graph remains random because non ACS structures are not robust [12]. This phase continues on average for a time $\tau_{a}=1 / p^{2} s$ until at some graph update a small ACS appears by chance and the growth phase begins (in Fig. 1, $\lambda_{1}$ jumps from zero to one at that very time step, and in general, at the beginning of every growth phase). A small ACS is robust. ( $i v)$ implies that members of the ACS do well populationally compared to species outside it, hence the latter are replaced in subsequent graph updates. When the new species receives a link from the existing dominant ACS, the latter typically expands and $s_{1}$ increases. This growth and self-organization continues over a timescale $\tau_{g} \ln s$ where $\tau_{g}=1 / p$ until the dominant ACS spans the entire graph and $s_{1}$ becomes equal to $s$ (see Fig. 3) [6, 10, 12]. That marks the beginning of the organized phase. Note that the entire graph in Fig. 3 is an ACS. In a fully spanned ACS the least populated species must be a member of the ACS. Now competition between members of the dominant ACS becomes important and can lead to fragility and rupture of the organization.

Let us define a crash as a graph update event $n$ for which $\Delta s_{1}(n)<-s / 2$, i.e., an event in which a significant number (arbitrarily chosen as $s / 2$ ) of the species go extinct. In runs with $s=100, p=0.0025$ totaling 1.55 million iterations we observed 701 crashes. It is evident from Fig. 1 that crashes typically take place at or near $\lambda_{1}=1$. This can be understood by taking a closer look at the structure of the dominant ACS.

The dominant ACS consists of a 'core' and a 'periphery'. The core of a dominant ACS is the maximal subgraph, $Q$, from each of whose nodes all nodes of the dominant ACS can be reached along some directed path. The rest of the dominant ACS is its periphery. For an example see Fig. 3. When the dominant ACS consists of two or more disjoint subgraphs the above definition applies to each component separately [17]. This distinction between core and periphery is useful in the context of the above dynamics. For example, the ratios of $X_{i}$ values of the core nodes are unchanged if any periphery node or link is removed from the dominant ACS, but removing or adding any node or link to the core in general changes all $X_{i}$ ratios. For any subgraph $A$ define $\lambda_{1}(A)$ to be the largest eigenvalue of the submatrix of $C$ corresponding to $A$. Then, it can be shown that $\lambda_{1}(Q)$ is the same as the largest eigenvalue of the whole graph, $\lambda_{1}$. The core (of each component) is an irreducible subgraph (i.e., one which contains at least two nodes and a directed path from each of its nodes to each of its other nodes). It follows from the Perron-Frobenius theorem that if some links are added to the core (with possibly additional nodes) $\lambda_{1}$ increases, and if removed from the core, $\lambda_{1}$ decreases. Thus $\lambda_{1}$ is a measure of the core size and multiplicity of pathways or 'redundancy' within it. $\lambda_{1}=1$ corresponds to the case where the core (of every disjoint component of the dominant ACS) has exactly one cycle. Such a core has no internal redundancy; the removal of any link from it will cause the ACS property (of that component) to disappear.

This is one, purely graph theoretical, reason why the organization is fragile in the vicinity of $\lambda_{1}=1$. Another reason is dynamical: when $\lambda_{1}>1$ the core nodes are better protected against selection by virtue of their larger populations, whereas at $\lambda_{1}=1$ they are more vulnerable. The reason is as follows: Since $\mathbf{X}$ is an eigenvector of $C$ with eigenvalue $\lambda_{1}$, when $\lambda_{1} \neq 0$ it follows that for nodes that belong to the dominant ACS, $X_{i}=\left(1 / \lambda_{1}\right) \sum_{j} c_{i j} X_{j}$. In particular, if a node $i$ of the dominant ACS has only one incoming link (from the node $j$, say) then $X_{i}=X_{j} / \lambda_{1}$, i.e., $X_{i}$ is 'attenuated' with respect to $X_{j}$ by a factor $\lambda_{1}$. The periphery of an ACS is a tree like structure emanating from the core, with most nodes having a single incoming link, for small $p$. Consider for example Fig. 3, in which the entire graph is an ACS with $\lambda_{1}=1.31$, and focus in particular on the chain of nodes $44 \rightarrow 45 \rightarrow 24 \rightarrow 29 \rightarrow 52 \rightarrow 89 \rightarrow 86 \rightarrow 54 \rightarrow 78$. The farther down such a chain a periphery node is, the lower is its $X_{i}$ because of the cumulative attenuation. For such an ACS with $\lambda_{1}>1$ the 'leaves' of the periphery tree will typically be the species with least $X_{i}$ (and node 78 in Fig. 3 is one such). However, when $\lambda_{1}=1$ there is no attenuation. Periphery nodes will not have lower $X_{i}$ than core nodes and some may have higher if they have more than one incoming link. Thus at $\lambda_{1}=1$ the core is not protected and in fact will always belong to $\mathcal{L}$ if the ACS spans the graph. $\lambda_{1}$ is known to be of significance in other complex systems as well [18 21].

We now present evidence that crashes are indeed due to changes in the structure of the core. Define the core overlap, denoted $O v\left(C, C^{\prime}\right)$, between two graphs $C$ and $C^{\prime}$ (whose nodes are labeled) as the number of common links in the cores $Q$ and $Q^{\prime}$ of their dominant ACSs (i.e., the number of ordered pairs of nodes $(i, j)$ such that $Q_{i j}$ and $Q_{i j}^{\prime}$ are both non-zero.) If either $C$ or $C^{\prime}$ does not have an $\mathrm{ACS}, \mathrm{Ov}\left(C, C^{\prime}\right)$ is by definition zero. A graph update event at time $n$ will be called a core-shift if $O v\left(C_{n-1}, C_{n}\right)=0\left(C_{n}\right.$ is the graph at time $\left.n\right)$. Fig. 4 shows that most (612) of the 701 crashes were core-shifts. (If a crash is defined as an event in which more than $90 \%$ 
of the species become extinct, then there are 235 crashes in these runs of which 226 are core-shifts.) Of the remaining 89 crashes 79 were 'partial core-shifts' and 10 were events in which the core remained unchanged.

In the 612 core-shifts, the average number of incoming plus outgoing links is 2.27 for all nodes in the graph, 2.25 for the node that is hit and 1.25 for the new node. Thus the nodes whose exchange causes the crash are not excessively rich in links (and the hit node is always the least populated). 'Nondescript' nodes such as these cause system wide crashes because of their critical location in a small core (the average core size at the 612 core-shifts is 6.3 nodes) that is responsible for the coherence and sustenance of the whole network 22. Core-shifts in which the ACS is completely destroyed typically cause the largest damage (of 612 core-shifts these are 136 in number, with $\left.\left|\Delta s_{1}\right|=98.2 \pm 1.2\right)$. The remaining 476 in which an ACS exists after the core-shift have $\left|\Delta s_{1}\right|=75.0 \pm 14.2$. The former constitute an increasing fraction of the crashes at smaller $p$ values, causing the upturn in $P\left(\Delta s_{1}\right)$ at large negative $\Delta s_{1}$ for small $p$ (Fig. 2).

Let $\tau_{s}$ denote the time for which the system stays in the organized phase until a core-shift occurs. $\tau_{s}$ increases with $p$ but its quantitative dependence on $p$ and $s$ remains an open question. After a crash if there is no ACS the graph usually becomes a random graph in order $s$ time steps. It takes on average $\tau_{a}=1 / p^{2} s$ time steps before a new ACS forms [6]. Once an ACS appears it grows exponentially across the graph on a time scale $\tau_{g}=1 / p$. After crashes in which an ACS survives the recovery time scale is just $1 / p$. The asymmetry between positive and negative changes in $s_{1}$ is a natural consequence of different processes being involved in the two cases.

It is characteristic of natural evolution that as different structures arise in the system the nature of the selective pressure on existing structures, and hence their effective dynamics, changes. In the present system we likewise see an effectively random graph evolution when there is no ACS, a self-organizing growth phase when an ACS, a small cooperative and hence robust structure, arises, and competition within the ACS resulting in its eventual fragility when a fully autocatalytic graph is formed. A different system in which the selective pressures and dynamics change as new structures arise is discussed in 23. Robust yet fragile structures also arise in highly designed systems 24]. In the present model the appearance of different structures dynamically generates different time scales: $\tau_{a}$ in the random phase, $\tau_{g}$ in the growth phase, and the survival time of the core, $\tau_{s}$, in the organized phase. These multiple structures and timescales arise endogenously, i.e., they are all consequences of the same underlying dynamical rules.

We thank O. Narayan for a discussion and drawing our attention to [13]. S.J. acknowledges the Associateship of Abdus Salam International Centre for Theoretical Physics, Trieste, and hospitality of the Max Planck Institute for Mathematics in the Sciences, Leipzig. This work was supported in part by a grant from the Department of Science and Technology, Government of India.

[1] M. E. J. Newman and R. G. Palmer, www.arXiv.org/ abs/adap-org/9908002.

[2] B. Drossel, www.arXiv.org/abs/cond-mat/0101409.

[3] R. N. Mantegna and H. E. Stanley, An introduction to econophysics: Correlations and complexity in finance (Cambridge Univ. Press, 1999).

[4] J.-P. Bouchaud, www.arXiv.org/abs/cond-mat/0008103.

[5] A. Johansen and D. Sornette, www.arXiv.org/abs/condmat/0010050.

[6] S. Jain and S. Krishna, Phys. Rev. Lett. 81, 5684 (1998).

[7] R. J. Bagley, J. D. Farmer and W. Fontana, in Artificial Life II, edited by C. G. Langton, C. Taylor, J. D. Farmer and S. Rasmussen, (Addison Wesley, Redwood City, 1991), p. 141.

[8] S. A. Kauffman, The Origins of Order (Oxford Univ. Press, 1993).

[9] W. Fontana and L. Buss, Bull. Math. Biol. 56, 1 (1994).

[10] S. Jain and S. Krishna, Computer Physics Comm. 121122, 116 (1999).

[11] P. Bak and K. Sneppen, Phys. Rev. Lett. 71, 4083 (1993).

[12] S. Jain and S. Krishna, Proc. Natl. Acad. Sci. (USA) 98, 543 (2001).

[13] A similar phenomenon for earthquake models albeit with a different mechanism is observed in J. M. Carlson and J. S. Langer, Phys. Rev. A 40, 6470 (1989).

[14] M. Eigen, Naturwissenschaften 58, 465 (1971).

[15] S. A. Kauffman, J. Cybernetics 1, 71 (1971).

[16] O. E. Rossler, Z. Naturforschung 26b, 741 (1971).

[17] Some rarely occurring ACS structures which do not alter our main conclusions need a more general definition of core and periphery (to be discussed elsewhere).

[18] L. Laloux, P. Cizeau, J.-P. Bouchaud, and M. Potters, Phys. Rev. Lett. 83, 1467 (1999).

[19] V. Plerou, P. Gopikrishnan, B. Rosenow, L. A. N. Amaral, H.E. Stanley, Phys. Rev. Lett. 83, 1471 (1999).

[20] I. J. Farkas, I. Derenyi, A.-L. Barabasi and T. Vicsek, www.arXiv.org/abs/cond-mat/0102335.

[21] K.-I. Goh, B. Kahng and D. Kim, www.arXiv.org/ abs/cond-mat/0103337.

[22] For a more detailed mechanism and classification of coreshifts, see S. Jain and S. Krishna, unpublished.

[23] M. D. Cohen, R. L. Riolo and R. Axelrod, www.santafe.edu/sfi/publications/Abstracts/99-01002abs.html.

[24] J. M. Carlson and J. Doyle, Phys. Rev. E 60, 1412 (1999).

\section{Figure legends}

Figure 1. The number of populated species, $s_{1}$ (continuous line), and the largest eigenvalue of $C$ (whose significance is discussed later in the text), $\lambda_{1}$ (dotted line), versus time, $n$. The $\lambda_{1}$ values shown are 100 times the actual $\lambda_{1}$ value. Runs shown have $s=100$, and (a) $p=0.001$, (b) $p=0.0025$ and (c) $p=0.005$. 
Figure 2. Probability distribution of changes in the number of populated species. $P\left(\Delta s_{1}\right)$ is the fraction of time steps in which $s_{1}$ changes by an amount $\Delta s_{1}$ in one time step in an ensemble of runs with $s=100$ and $p=0.001,0.0025,0.005$. Only time steps where an autocatalytic set initially exists are counted.

Figure 3. The structure of the graph at $n=2885$ for the run in Fig. 1b, when the dominant ACS spanned the entire graph for the first time. Node numbers $i$ from 1 to 100 are shown in the circles representing the nodes. Black circles correspond to nodes in the 'core' of the ACS, and grey to the 'periphery', defined in the text.

Figure 4. Frequency, $f$, of core overlaps in crashes for runs with $s=100, p=0.0025$. The $x$-axis displays the value of $O v\left(C_{n-1}, C_{n}\right)$ in crashes (i.e., in the 701 events with $\left.\Delta s_{1}(n)<-50\right)$.
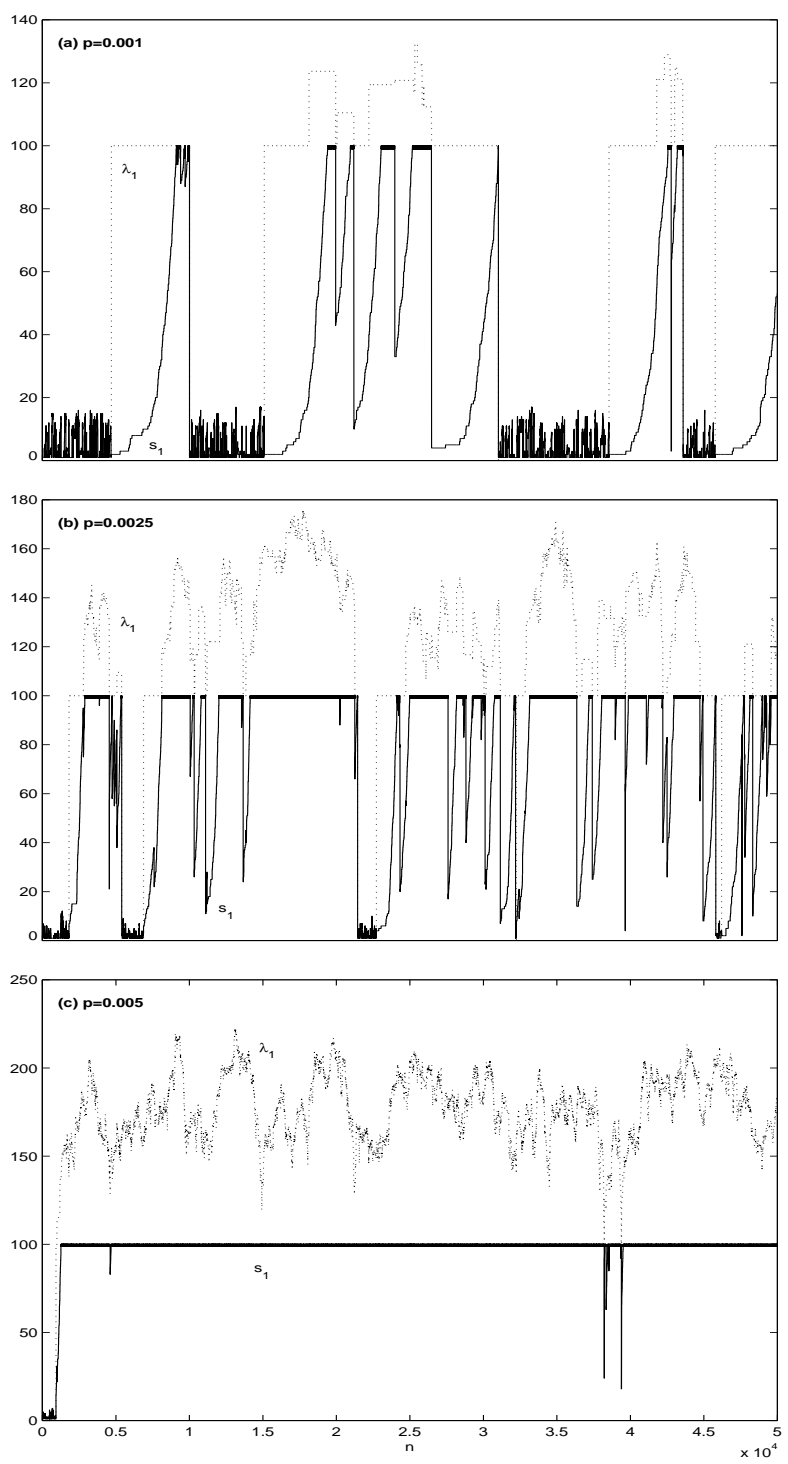

Figure 1.

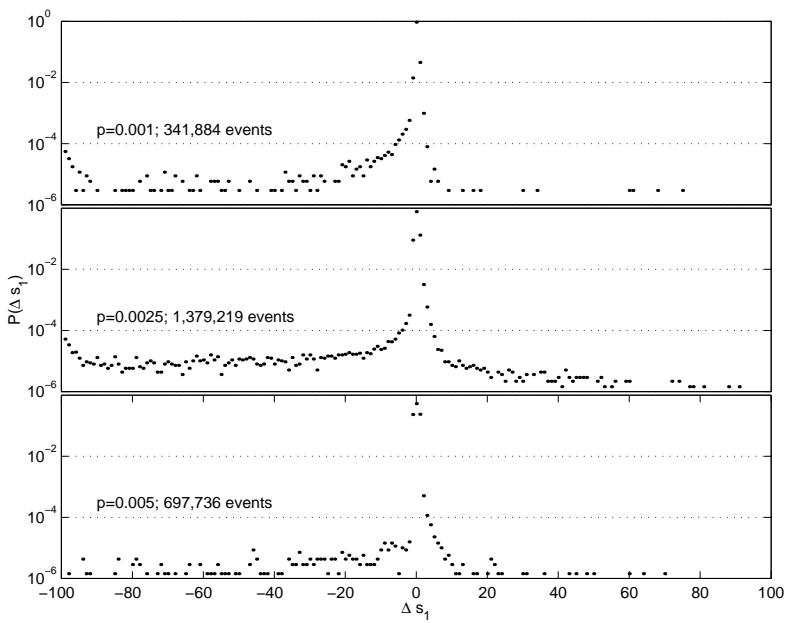

Figure 2.

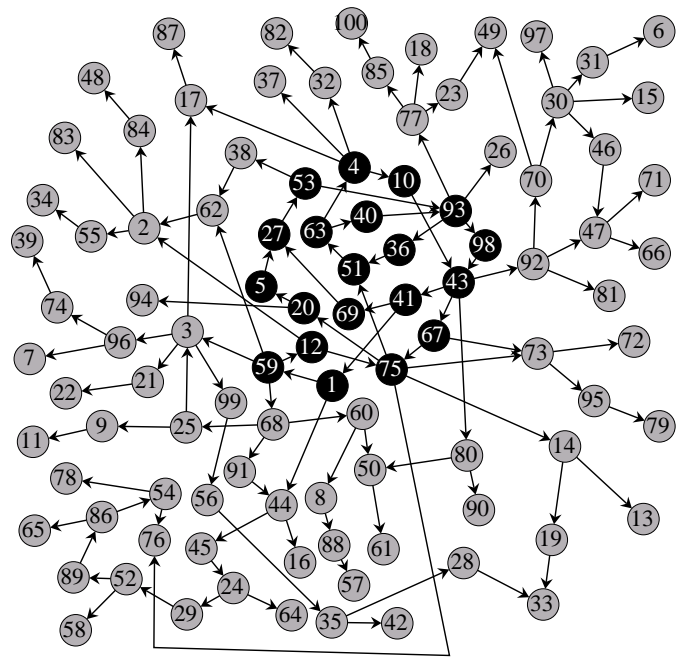

Figure 3.

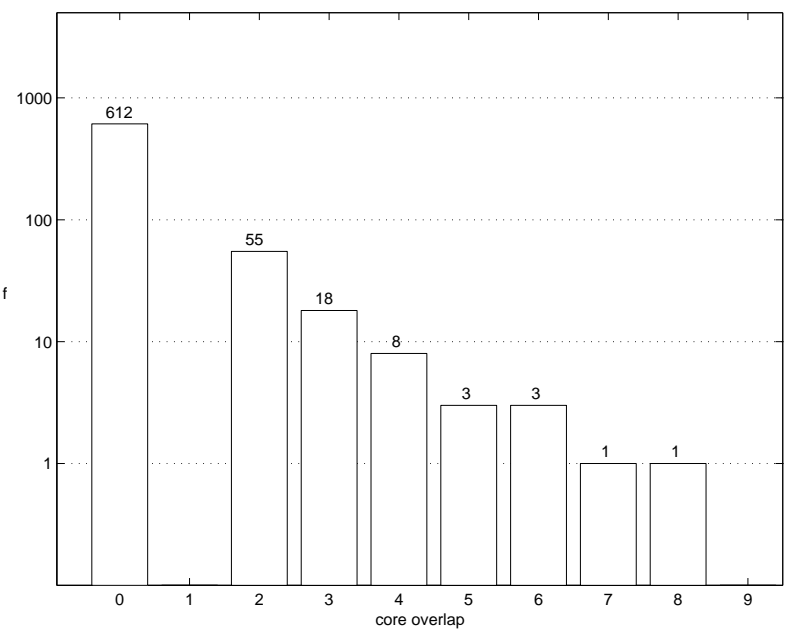

Figure 4. 